\title{
Evolución del cooperativismo de consumo
}

\author{
Alejandro Martínez Charterina \\ Catedrático de la Universidad de Deusto \\ Presidente de la Asociación Internacional de Derecho Cooperativo
}

Recibido: $\quad$ 16.06.11

Aceptado: 05.07 .11

Sumario: I. La cooperativa de consumo en el origen del cooperativismo moderno. II. La cooperativa de Rochdale. III. Los principios de Rochdale. IV. Comienzo del cooperativismo de consumo en España. V. Grandes cambios en la evolución del cooperativismo de consumo: 5.1. El crecimiento y asociacionismo; 5.2. La pluralidad de actividades; 5.3. Los cambios en los principios cooperativos; 5.4. La competencia y la velocidad del cambio; 5.5. La legislación administrativa y cooperativa; 5.6. El consumidor y su defensa. VI. Un modelo particular: la cooperativa Eroski. VII. Elementos destacados del modelo: 7.1. Venta a terceros y obtención de dimensión competitiva; 7.2. Trabajadores socios de trabajo y dos comunidades que conviven y deciden conjuntamente; 7.3. Desaparición del retorno y establecimiento de una filosofía social sobre los excedentes; 7.4. Defensa del consumidor. VIII. La evolución continúa. IX. Alternativas. X. Conclusión. XI. Bibliografía.

Resumen: La cooperativa de consumo se sitúa en el origen del cooperativismo moderno. A lo largo del tiempo se han producido muchos cambios, en algunos momentos muy intensos y muy rápidos, y han afectado a la marcha y existencia de las cooperativas de consumo. Estas han evolucionado para profundizar en su servicio y mantener su sentido. Se examina con detenimiento el caso de la cooperativa Eroski, que con origen en el País Vasco extiende su actividad y se sitúa entre las grandes sociedades de distribución siguiendo un nuevo modelo, y se fija la atención también en una cooperativa local, de barrio, la Park Slope Food Coop de Brooklyn, como una alternativa. En ambos casos el consumidor, su formación y la defensa de sus derechos son de la máxima importancia.

Palabras clave: cooperativas de consumo, evolución del cooperativismo de consumo, derechos del consumidor.

Abstract: The origins of modern cooperativism can be found in the consumer cooperative. Many changes have taken place over time, some of which have been profound and rapid, affecting the way consumer cooperatives work and their very existence. They have evolved to give more complete service and keep their original purpose. The case of the Eroski cooperative is studied in 
depth.Eroski originated in the Basque Country and expanded its business activities to become one of the big distributors following a new model. A local neighbourhood cooperative, the Park Slope Food Coop in Brooklyn, is studied as an alternative to the former case. In both cases, consumers, their awareness and protection of their rights are of utmost importance.

Key words: consumer cooperatives, evolution of consumer cooperativism, consumer rights. 


\section{La cooperativa de consumo en el origen del cooperativismo moderno}

Las primeras cooperativas modernas ${ }^{1}$ están relacionadas con la búsqueda de la satisfacción de las necesidades básicas de la población más desfavorecida. Cancelo y Dacosta lo vinculan a la defensa y mantenimiento de la capacidad adquisitiva de los salarios en un contexto comercial desfavorable a los trabajadores².

Es en Gran Bretaña donde se identifican las primeras de ellas desde la segunda mitad del siglo XVIII, es decir desde el comienzo del proceso de industrialización y en sintonía con el problema social que se iba produciendo.

Alicia Kaplan de Drimer y Bernardo Drimer citan las organizadas por obreros de los Astilleros de Woolwich y Chatham en 1760, en Londres y sus proximidades, que pretendían el abastecimiento de harina, la base de la alimentación en aquellas comunidades.

Del mismo modo, tratando de aprovisionarse de útiles de trabajo se forman en Escocia las de los tejedores de Fenwick en 1769 y de Goban en $1777^{3}$.

En la primera parte del siglo XIX, se sitúa la Cooperative Trading Association en Brighton, fundada en 1827 por William King, así como las aproximadamente trescientas cooperativas de consumo que, bajo su inspiración se pondrán en marcha en aquellos años en los que el mismo Dr. King publicaría el periódico mensual The cooperator ${ }^{4}$, entre mayo de 1828 y agosto de 1830, y que se reunieron en diversos Congresos entre 1831 y 1835 .

También en este tiempo nacen cooperativas de consumo en Lyon (Francia). En primer lugar, en 1928, un grupo de trabajadores, jefes de taller, constituyeron Le devoir mutuelle para realizar compras de bienes

1 Dejadas atrás las formas precooperativas y demás aprovechamientos y usos colectivos a través de los cuales las personas han organizado sus esfuerzos conjuntos y solidarios en muchos lugares a lo largo de la Historia.

2 Antonio Cancelo: «Pasado, presente y futuro del cooperativismo de consumo en España» (1992), p. 14; Constan Dacosta: «Eroski, una adaptación al cambio» (1991), p. 18.

3 Alicia Kaplan de Drimer y Bernardo Drimer: Las cooperativas. Fundamentos, historia, doctrina (1983), p. 226.

4 El periódico llevaba en su encabezamiento la frase «Knowledge and union are power; Power, directed by knowledge, is happiness; Happiness is the end of creation», y cada ejemplar se componía de cuatro páginas que redactaba William King en su totalidad. 
de consumo en común 5 , y, pocos años después, en 1835, por iniciativa de Michel Derrion se pondrá en marcha Le commerce véridique et social, cooperativa ésta en la que Charles Gide veía los rasgos principales que algunos años después vendrían a caracterizar la cooperación rochdaleana6.

También el cooperativismo de trabajo inicia su andadura en estos primeros tiempos en los que Philippe Bouchez con un grupo de artesanos pone en marcha una cooperativa de ebanistería en 1832, de muy corta duración, y poco después, en 1834, otra cooperativa de joyería, la Association chrétienne des bijoutiers en doré, que duró hasta $1873^{7}$.

Y en los años siguientes se registran dos cooperativas para la distribución de pan, la Boulangerie Mutuelle de Ginebra (Suiza), en 1837, y la Boulangerie Véridique de París, en $1838^{8}$.

\section{La cooperativa de Rochdale}

La cooperativa de Rochdale, The Rochdale Society of Equitable Pioneers, se fundó en 1844 .

La que consideramos con la doctrina como el punto de partida del cooperativismo moderno vino precedida de por lo menos las cooperativas que acabamos de mencionar, lo que pone de manifiesto que no fue cronológicamente la primera y que su primacía está determinada por las aportaciones que realizó al cooperativismo posterior.

En efecto, la cooperativa de Rochdale recogió a través de sus Estatutos los principios y reglas de funcionamiento de una cooperativa de consumo. Estas pautas de conducta iban a ser copiadas por las cooperativas posteriores, de forma que acabarían siendo reconocidas como la primera declaración de los principios cooperativos, y constituyéndose, en consecuencia, como elemento calificativo y diferenciador de la empresa cooperativa9.

5 También actuaba como una especie de agrupación sindical para canalizar sus reivindicaciones.

6 Denis Bayon: Le commerce véridique et social de Michel-Marie Derrion. Lyon 1835-1838 (2002), pp. 21 y 22.

7 Además de los Drimer, puede verse en Gromoslav Mladenatz, Historia de las doctrinas cooperativas (1969), pp. 51 y 52.

8 También citan las dos panaderías en Maurice Colombain: Las cooperativas (1967), p. 7.

9 En este sentido Gromoslav Mladenatz: o.c., p. 65. En el mismo sentido en el centenario de la cooperativa de Rochdale, Bernardo Delom insiste en estas razones para 
Por otro lado, la cooperativa de Rochdale sigue en activo desde su fundación. Esto contrasta con la vida efímera de las cooperativas anteriores a las que nos hemos referido (con excepción de la Association chrétienne des bijoutiers en doré, que duró casi cuarenta años), que por diversos motivos, en ocasiones circunstanciales como la incidencia de la crisis económica, caso de Le commerce véridique et socia/ ${ }^{10}$, o por las dificultades para llevar a cabo una gestión con éxito por las limitaciones en cuanto al número de asociados y la falta de estímulos para éstos, lo que parece sucedió en las cooperativas de consumo promovidas por el Dr. King ${ }^{11}$, no tuvieron muchos años de actividad.

La cooperativa de Rochdale se constituyó en ese mismo contexto en el que los trabajadores soportan el cúmulo de dificultades que se ha sintetizado en el denominado «problema social» derivado del proceso de industrialización, al que nos hemos referido en el epígrafe anterior.

Algunos tejedores de Rochdale, barrio de Manchester, que acaban de fracasar en la consecución de un aumento de salario que pretendían y que consideraban justificado por el aumento de los beneficios de la industria textil a finales de 1843, deciden mejorar sus condiciones de vida de otra manera12.

El resultado de la reflexión y el debate sostenido fue la constitución de la Rochdale Society of Equitables Pioneer por los 28 tejedores en octubre de 1844, para poner en actividad un almacén cooperativo de consumo, cuya apertura se llevó a cabo el 21 de diciembre siguiente, en el piso bajo de una casa en un callejuela de poca importancia (Toad Lane) que arrendaron con esa finalidad.

A pesar de la modestia con la que comenzó su actividad la cooperativa, con gran esfuerzo lograron reunir la cifra de 28 libras de capital (que no estaba siquiera desembolsado totalmente al principio), sus objetivos pretendían un mejoramiento decidido de sus condiciones de vida, a través de las compras en el almacén de alimentos, ropas y otras provisiones, la adquisición de viviendas para sus miembros interesados en ello, la fabricación de determinados artículos para dar trabajo a los miembros desempleados, la adquisición de tierras para explotarlas también por parte de los socios desempleados... todo ello como tránsito hacia la creación de una colonia autosuficiente... También se pretendía ayudar a otros a constituir colonias similares. Del mismo modo, y en

considerarla la iniciadora del movimiento cooperativo mundial, en la «Introducción» al libro de Jorge Jacobo Holyoake, Historia de los pioneros de Rochdale (1973), p. 11.

10 Denis Bayon (2002): o.c., pp. 36 ss.

11 Gromoslav Mladenatz (1969): o.c., p. 35.

12 Georges Jacob Holyoake (1989): o.c., pp. 9 y 10. 
otro orden de cosas se abriría un salón de templanza para fomentar la sobriedad entre los asociados ${ }^{13}$.

Cierto que no todo se pudo llevar a cabo, y que algunos intentos fracasaron, pero se puso en marcha el cooperativismo de consumo que ha llegado hasta nuestros días, y, como se ha señalado, las bases del cooperativismo moderno y del movimiento cooperativo internacional.

\section{Los principios de Rochdale}

Se han realizado varias formulaciones de los principios de Rochdale, que no se encuentran enumerados y dispuestos ordenadamente en los Estatutos de la Sociedad.

Paul Lambert ${ }^{14}$ realiza una de estas formulaciones considerando los Estatutos de 1844, las enmiendas a los Estatutos de 1845, y varios acuerdos asamblearios posteriores, en la que propone los siguientes principios:

- el control democrático;

- la libre adhesión;

— el interés limitado del capital;

- la distribución de los beneficios en proporción a las compras realizadas;

- las compras y ventas al contado;

- la pureza y calidad de los productos;

- la promoción de la educación entre los miembros;

- la neutralidad política y religiosa;

- las ventas a precio de mercado;

- la adhesión voluntaria;

- la devolución desinteresada del activo neto en el caso de disolución de la sociedad;

- la aspiración de cooperativizar la organización económica y social del mundo.

Merece la pena destacar algunos de estos principios en el contexto en el que se pone en marcha la cooperativa de Rochdale. Los trabajadores industriales, que se han desplazado del campo a las ciudades que crecen a ritmo vivo al tiempo que se van instalando las grandes fábricas mecanizadas, son presa de múltiples problemas.

13 Laws and objets of the Rochdale Society of Equitable Pioneers: enrolled according to the Acts, $10^{\text {th }}$, George $N$, and $4^{\text {th }}$ and $5^{\text {th }}$, William IV (1844), p. 3.

14 Paul Lambert: La doctrina cooperativa (1970), p. 57. 
En lo que se refiere a la distribución de mercancías, estas se vendían en las tiendas de barrio, siguiendo un modelo de competencia imperfecta que establecía que los trabajadores compraban lo que les vendían sin asegurar calidades, sin corrección de los pesos y medidas, y con la aparente ventaja de la venta a crédito que vinculaba al trabajador y su familia con la tienda.

La cooperativa de Rochdale se empeña en llevar a cabo las compras y ventas al contado, en vender a precio de mercado, pero asegurando la pureza y calidad de los productos, en distribuir los beneficios en atención a las compras efectuadas, desvinculando el beneficio del capital que recibirá como retribución un interés limitado, y promocionando la educación entre sus miembros. A la cooperativa se accede libre y voluntariamente, lo que lleva implícito el deseo de ser socio y de participar activamente en la marcha y gestión democrática, cada socio un voto, de la cooperativa.

\section{Comienzo del cooperativismo de consumo en España}

El cooperativismo de consumo se extiende a partir de Rochdale por toda Europa, al tiempo que se va estableciendo el cooperativismo de trabajo, el crédito cooperativo, el cooperativismo agrícola, y la prestación de determinados servicios, como los seguros o las viviendas, en forma cooperativa, poniendo de manifiesto, como advierte la Declaración de la Alianza Cooperativa Internacional sobre la Identidad Cooperativa, en la parte titulada «En los albores del siglo XXI. Las Cooperativas ayer, hoy y mañana», que avanzando el siglo XIX "parecía haber pocos límites a las posibilidades de la acción cooperativa» ${ }^{15}$.

Con retraso respecto a otros países europeos, la cooperativa de consumo llega a España a través de la Económica Palafrugellense, fundada en Palafrugell, provincia de Gerona en 186516, bajo la inspiración del pensamiento de Fernando Garrido.

Al País Vasco llegaron las cooperativas todavía más tarde, finales del siglo XIX, siendo las primeras las de consumo. En Vizcaya la Socie-

15 A.C.I., Declaración...(1986), p. 75.

16 Si bien se habla de algunas anteriores, una de consumo en Barcelona hacia 1840, otra en Valencia, El compañerismo, en 1856. Sobre estos inicios discutidos puede verse Leyre Arrieta et al.: El movimiento cooperativo en Euskadi 1884-1936 (1998), pp. 3436. En particular, se cita la cooperativa valenciana La proletaria y su constitución en 1850, como primera cooperativa de consumo, en «Consumer and User Co-operatives in Spain», en Review of International Co-operation (1990), p. 6. 
dad Cooperativa de Obreros de Baracaldo se constituyó en 1884 con trabajadores de Altos Hornos de Bilbao, y en 1887 la Cooperativa de Consumo de Sestao, para los trabajadores de la empresa La Vizcaya. En Álava la primera cooperativa fue la Unión Obrera de Araya, de 188617, también vinculada a una empresa, en este caso la Fábrica de Hierro, Ferrería y Talleres de Herraje de Araya. Y en Guipúzcoa la primera cooperativa de consumo parece ser La Sociedad Cooperativa de Obreros, fundada en Eibar en $1893^{18}$.

Estas primeras cooperativas de consumo del País Vasco se caracterizan por su vinculación a las empresas, llegando a tener muchas un patronato empresarial. Entre ellas se pueden citar también la Cooperativa La Arboleda de la Sociedad Orconera, la de las minas de Galdames, la de la Compañía del Cadagua de Aranguren, la de la empresa La Basconia de Basauri, la de los Ferrocarriles de Santander a Bilbao, las de la Papelera Española, en Vizcaya, y en Guipúzcoa la de la Unión Cerrajera de Mondragón, y la Cooperativa de la Fábrica de Cemento de Añorga, entre otras ${ }^{19}$.

\section{Grandes cambios en la evolución del cooperativismo de consumo}

La historia nos ofrece una sucesión de acontecimientos a medida de que transcurre el tiempo que revelan que en ocasiones conseguimos nuestros propósitos, otras veces tenemos que renunciar a ellos en todo o en parte y siempre operan acciones ajenas a nosotros mismos que nos favorecen o nos perjudican al tiempo que nuestras propias acciones afectan también a otros. En suma estamos sometidos a cambios en el marco de nuestras propias circunstancias, y es en ese contexto de cambio en el que nos movemos hacia adelante, en el que se produce nuestra evolución. El cambio como una constante y la respuesta y adaptación al cambio determinan, por tanto, la evolución del cooperativismo de consumo.

Quisiera detenerme en algunos de los elementos de cambio que se hecho presentes en el cooperativismo de consumo, en ocasiones con-

17 Este año, por tanto, celebra su 125 aniversario.

18 Así, en Leyre Arrieta (1998): O.C., pp. 117 ss. Se presta a confusión la consideración de una primera cooperativa anterior en Guipúzcoa en Ermua en 1887, toda vez que Ermua es Vizcaya, en Concepción Roussell y Roberto Albóniga: Historia de las cooperativas de consumo vascas (1994), p. 33.

19 Leyre Arrieta (1998): o.c., p. 123. 
dicionándolo, presentándose como un reto, y en otras como una oportunidad, pero en todo caso compartiendo el caminar de esas cooperativas y de sus miembros.

Me referiré en concreto a:

- el crecimiento y asociacionismo;

- la pluralidad de actividades;

- los cambios en los principios cooperativos;

- la competencia y la rapidez del cambio;

- la legislación administrativa y cooperativa;

- el consumidor y su defensa.

\subsection{El crecimiento y asociacionismo}

La cooperativa de Rochdale en su apertura sólo disponía para vender de pequeñas cantidades de manteca, azúcar, harina de trigo y avena ${ }^{20}$. Tres meses después vendía también té y tabaco, dos años más tarde carne, y al cabo de cinco años se vendían también libros y diarios. Siete años después el almacén abría sus puertas durante todo el día, incluso los sábados, para atender a sus socios.

Después de nueve años, en 1853, la cooperativa contaba con seis secciones: comestibles, carnicería, tienda, mercería, calzado y sastrería, e iniciaba la venta al por mayor ${ }^{21}$.

En la medida en la que la cooperativa resultaba de interés para las personas, era cada vez mayor el número de sus asociados, y el crecimiento en número conducía a una mejor atención a sus socios proporcionándoles productos más variados, atendiéndoles durante más tiempo, ofreciéndoles más oportunidades educativas, etc.

Una de las palancas que vendrían a impulsar el cooperativismo con carácter general ha sido precisamente el asociacionismo. Las cooperativas han tratado de afianzar y extender su presencia a través de un movimiento cooperativo constituido de abajo hacia arriba. El movimiento cooperativo, dicen los Drimer, «expresa una tendencia o esfuerzo común encaminado hacia un propósito específico, que consiste precisamente en la difusión y consolidación de las entidades cooperativas como forma ideal de organización....»22.

20 Puede verse en Georges Jacob Holyoake (1989): o.C., pp. 17ss.

21 G.J. Holyoake (1989): o.c., p. 75.

22 Kaplan de Drimer y Drimer (1981): o.c., p. 39. 
La asociación de personas que es la cooperativa se prolonga en la asociación de las cooperativas entre sí, es la conclusión de un proceso inacabado23.

El movimiento trasciende los niveles locales y regionales para llegar al conjunto de la nación y al nivel internacional, habiéndose fundado la Alianza Cooperativa Internacional, que recoge las cooperativas de todo el mundo a través de sus federaciones y confederaciones, en el año 1895.

Aún cuando el movimiento cooperativo tenía un carácter fundamentalmente representativo, las cooperativas de consumo podían también beneficiarse de las compras realizadas a través de centrales establecidas con esa finalidad, e incluso podían organizar directamente la producción de algunos artículos.

Si los movimientos cooperativos inglés y francés y otros europeos estaban formados y preparados para impulsar un movimiento universal a través de la Alianza Cooperativa Internacional a finales del siglo XIX, en España la primera reunión de cooperativas de carácter regional, primer germen de un futuro movimiento cooperativo, tuvo lugar en 1899. Esta reunión se convirtió en interregional, I Congreso Cooperatista Catalano-Balear, por la participación de tres cooperativas de las Islas Baleares.

El I Congreso Nacional de Cooperativas tuvo lugar en Barcelona en 1913. A partir del mismo se inició la constitución de un movimiento federativo en el que se incluye la Unión de Cooperativas del Norte de España en 1914, con cooperativas de Vizcaya sobre todo, Guipúzcoa, Álava, Santander, Burgos y Logroño, la Federación Regional Valenciana y la Federación Regional de Cataluña reconstituida.

En los siguientes Congresos que se fueron celebrando, y al tiempo que iba creciendo el movimiento cooperativo en las diversas regiones, fue calando la idea de reunirse en una Federación Nacional de Cooperativas, que finalmente se constituyó en 1929. Esta Federación, que agrupaba a 500 cooperativas y 200.000 socios, solicitó su ingreso en la Alianza Cooperativa Internacional24.

\subsection{La pluralidad de actividades}

La cooperativa de consumo está ligada en sus orígenes al suministro de bienes de primera necesidad, como hemos señalado anterior-

23 Alejandro Martínez Charterina: Análisis de la integración cooperativa (1990), pp. 16-18.

24 Leyre Arrieta et al. (1998): o.c., pp. 37-42. 
mente. Sin embargo en las cooperativas de consumo se reúnen personas que tratan de satisfacer sus necesidades de suministro de bienes y servicios a través de una empresa que, gestionada por los socios, les proporciona los bienes y servicios que precisan. Por ello, uno de los elementos de desarrollo se refiere a la pluralidad de actividades de la cooperativa de consumo ${ }^{25}$.

De este modo de los alimentos, se pasó al vestido y demás artículos necesarios para la familia, pero también a la enseñanza, consumo de enseñanza, a los servicios de lo relacionado con el ocio, a los servicios sanitarios, asistenciales, culturales,... teniendo en consideración, como señalan Ignasi Faura y Teresa Udina, que en el origen de la cooperativa de consumo sólo se encuentra una necesidad colectiva que espera una respuesta26.

Cada país con su legislación, al establecer las clases de cooperativas, agrupa en torno a las de consumo una pluralidad concreta. En el caso español, si atendemos a la clasificación que realiza la Confederación de Cooperativas de Consumidores y Usuarios (HISPACOOP), dentro de las cooperativas de consumidores y usuarios encontramos cooperativas de distribución alimentaria, que no sólo distribuyen alimentos, de cultura y educación, de sanidad y servicios, eléctricas y otras ${ }^{27}$.

Entre las cooperativas de distribución alimentaria se encuentran Eroski y la cooperativa Consum, con 2.367 puntos de venta la primera y 575 la segunda, junto a otras que sólo tienen un punto de venta ${ }^{28}$; en las de cultura y educación destaca la cooperativa Abacus, que distribuye libros, material escolar y juguetes educativos en 32 puntos de venta; en las de sanidad y servicios, SCIAS, cooperativa de usuarios de los servicios sanitarios propietaria del Hospital de Barcelona, que agrupa a 170.000 personas; la distribución eléctrica producida o adquirida por la cooperativa que recibían 46.573 socios en 2009; la gestión de residencias de ancianos (Cooperativa Pi i Sunyer), atención a disminuidos psíquicos (Cooperativa l'Estel), la gestión de servicios ciudadanos de gestoría (Cooperativa Gestió Veïnal de Barri),

25 Puede considerarse el planteamiento tradicional que entiende que las cooperativas lo son de consumo o de producción, de forma que los intereses se centraban en el consumo de bienes y servicios, por un lado, o en la producción de los mismos, por otro. En este sentido puede verse Iván Jesús Trujillo Díez: Cooperativas de consumo y cooperativas de producción (2000), pp. 27 a 30.

${ }^{26}$ Ignasi Faura y Teresa Udina: "La actividad de las cooperativas de consumidores», en Economía social. La actividad económica al servicio de las personas (s.f.), p. 149.

27 Remedios Aceña: "Cooperativas y consumidores: Los protagonistas de estos veinte años», en Cuadernos de las cooperativas de consumidores (2010), pp. 22 a 25.

28 Datos de 2009, en Remedios Aceña (2010): o.c., p. 23. 
entre otras, muestran la variedad de actividades que interesan a las cooperativas de consumo 29 .

\subsection{Los cambios en los principios cooperativos}

Los principios de funcionamiento de la cooperativa de Rochdale, como hemos indicado anteriormente, sirvieron de base de comportamiento a las cooperativas que se fueron constituyendo con posterioridad. A pesar de ello la Alianza Cooperativa Internacional, creada en 1895, no se preocupó por la identidad de las cooperativas hasta el X Congreso que tuvo lugar en Basilea el año 1921. En dicho Congreso resolvió que las cooperativas debían acomodar su conducta a los principios de Rochdale, como venían haciendo, y que esos principio, por consiguiente, determinarían la identidad de las cooperativas $^{30}$.

A partir de esta declaración la Alianza volvería sobre los principios cooperativos en tres nuevas ocasiones, tratando de ajustarlos precisamente a los cambios que se iban produciendo, de forma que la cooperativa se acomodara también a cada tiempo.

En el XV Congreso de París, en 1937, la Alianza estableció dos categorías de principios, unos primarios o principales, que entroncaban con la identidad de las cooperativas, la libre adhesión, el control democrático, la distribución de los excedentes en función de las compras realizadas, y el interés limitado al capital, y otros adicionales no imprescindibles aunque recomendables, la neutralidad política y religiosa, las compras y ventas al contado y la promoción de la educación. Han cambiado las cosas y las compras y ventas al contado ya no son esenciales en ausencia de los hábitos del consumo a crédito que a través de la cooperativa se querían evitar.

El XXIII Congreso de Viena, en 1966, reduce a seis los principios, todos ellos de igual importancia en la determinación de lo qué es una cooperativa, y de cómo debe comportarse. Libre adhesión, gestión democrática, interés limitado al capital, reparto de excedentes en propor-

29 Información y datos en Remedios Aceña (2010), Ignasi Faura y Teresa Udina (s.f.): o.c., pp. 150 y 151, e HISPACOOP: Cooperativas de consumidores y usuarios. Cómo empezar (2001), pp. 16 a 31.

30 Alejandro Martínez Charterina, «La cooperativa como empresa y los principios cooperativos tras el congreso centenario de la ACI de Manchester» (1996), p. 215. En este trabajo se presenta la evolución de los principios cooperativos hasta la declaración actual de los mismos, y baso en él la explicación de este punto. 
ción a las transacciones, educación, y el nuevo principio de cooperación entre cooperativas. La neutralidad se da por supuesta y las compras y ventas al contado han quedado superadas.

Sin embargo el principio sexto de intercooperación tiene la máxima importancia. Había sido reiteradamente solicitado ante la internacionalización y la dimensión creciente de las empresas que acentuaban la competencia y las situaciones monopolísticas, ante las que se quería poner de manifiesto que las cooperativas no sólo podían asociarse en términos representativos a través de las federaciones, destacando el aspecto social, sino también en términos económicos, destacando el aspecto empresarial.

Con ocasión del centenario de la Alianza Cooperativa Internacional, se revisó la identidad cooperativa en el Congreso de Manchester, en 1995. Además de definir la identidad en términos no sólo de principios sino también de valores o aspiraciones que los sostienen, introduce dos nuevos principios, el de autonomía e independencia de las cooperativas y el del interés de éstas por la comunidad.

Así se mantienen la libre y voluntaria adhesión, la gestión democrática, el interés limitado al capital y el reparto de excedentes fusionados en un tercer principio de participación económica de los socios, el nuevo de autonomía e independencia, la educación, formación e información, el sexto de cooperación entre cooperativas, y el séptimo, nuevo, de interés por la comunidad.

El principio de autonomía e independencia pretende reforzar ésta ante posibles situaciones de dependencia de poderes públicos o privados que puedan dificultar el ejercicio del control democrático de los socios, a partir de experiencias en algunos países en vías de desarrollo, y el último de interés por la comunidad pone de manifiesto una sensibilidad de las cooperativas por sus comunidades y por su desarrollo en forma sostenible y respetuosa con el medio ambiente.

Las cooperativas son entidades que se acomodan a su tiempo a través de unos principios o pautas de conducta que cambian adecuándose a la sociedad, pero que mantienen unos valores o aspiraciones permanentes que pretenden alcanzar a través de estos principios.

\subsection{La competencia y la velocidad del cambio}

Desde los años sesenta y setenta del siglo pasado en nuestro país, y seguramente algo antes en otros países desarrollados, se va a ir produciendo un conjunto de elementos de cambio impactantes para las coo- 
perativas de consumo, que podemos agrupar en torno a una idea de cambio en la competencia.

En efecto, la transformación del comercio se va a hacer presente a través de las nuevas formas de distribución comercial regidas por el principio de que vender mayor cantidad con un margen menor proporciona un mayor beneficio. Aparecen los autoservicios y supermercados, y después también los hipermercados, las grandes superficies, que irán superando el modelo de la tienda pequeña, de barrio o de localidad, en el que se asentaba la cooperativa de consumo tradicional ${ }^{31}$.

Esta evolución sintonizará con el fenómeno de la concentración empresarial, que viniendo de un tiempo anterior desembocará en las multinacionales de la alimentación y en formas monopolísticas del mercado.

Y esta situación novedosa vendrá a ser más profunda en los años siguientes a través de los cambios tecnológicos de una gestión informatizada y de la aplicación del marketing y sus técnicas de publicidad y promoción en particular ${ }^{32}$.

El cooperativismo de consumo deberá sobrevivir, en primer lugar, y quedar como alternativa a estos fenómenos de cambio, a esta competencia más poderosa.

En este contexto se pueden tener en consideración algunos datos de cómo cambia el mercado español en los sólo nueve años que van de 1980 a 1989:

\begin{tabular}{lrr}
\hline \multicolumn{1}{c}{ Cuota de mercado $\rightarrow$} & 1980 & 1989 \\
\hline Hipermercados & $7 \%$ & $20 \%$ \\
Supermercados & $17 \%$ & $42 \%$ \\
Autoservicios & $23 \%$ & $15 \%$ \\
Tradicionales & $53 \%$ & $23 \%$ \\
\hline
\end{tabular}

Fuente: A. Cancelo, «Pasado, presente y ...», o.c. (1992), p. 17.

31 Constantes referencias en Antonio Cancelo: "Cooperativismo y consumerismo», en Anuario de estudios cooperativos 1986, p. 210; "El cooperativismo ante las exigencias del mercado mundial», en Anuario de estudios cooperativos 1987, p. 83, «Posicionamiento del cooperativismo de consumidores vasco», en /l Congreso mundial vasco. Congreso de cooperativismo (1988), p. 319; «Pasado, presente y futuro...» (1992), o.c., p. 15; también en Constan Dacosta (1991): o.c., p. 19, que considera valorable la acción voluntarista de las cooperativas pequeñas junto a su incapacidad para avanzar hacia el futuro sólo con voluntarismo, y en Fermín Garmendia y Manolo González: Principios, pensamiento e innovación empresarial. La visión de Antonio Cancelo (2010), p. 30.

32 Concepción Roussell y Roberto Albóniga (1994): o.c., pp. 79 a 82. 
Vendrá, en consecuencia, y como respuesta de las cooperativas, un tiempo de reducción del número de cooperativas de consumo, mientras aumentan todos los demás parámetros comparativos:

\begin{tabular}{lcc}
\hline \multicolumn{1}{c}{ Año } & 1989 & 2009 \\
\hline Cooperativas asociadas & 450 & 128 \\
Socios consumidores & 645.000 & 2.853 .731 \\
Trabajadores & 5.490 & 59.578 \\
Facturación (millones $€$ ) & 718,20 & $10.282,55$ \\
Superficie $\left(\mathrm{m}^{2}\right)$ & 300.000 & 2.557 .327 \\
Puntos de venta & 665 & 3.097 \\
\hline
\end{tabular}

Fuente: Remedios Aceña, o.c. (2010), p. 21.

Esta evolución, que se refiere a las cooperativas de consumidores y usuarios españolas asociadas a HISPACOOP, es constante en el periodo de veinte años contemplados en la tabla completa de la que se han extraído los datos de los extremos del periodo de veinte años que se recogen en ella.

Junto al cambio de competencia, presencia de las multinacionales de distribución en un mundo internacionalizado primero y después globalizado, debe destacarse la velocidad del cambio. Es en un corto espacio de tiempo en el que se producen los cambios a los que tendrá que enfrentarse para sobrevivir la cooperativa de consumo de pequeña dimensión local, que toma sus decisiones de forma democrática pero con gran lentitud, lo que juzga Cancelo «...suicida en situaciones de cambio acelerado... $\rangle^{33}$.

\subsection{La legislación administrativa y cooperativa}

Otro de los elementos de consideración en el tiempo es, a mi entender, el progreso en la legislación administrativa que trata de proteger al consumidor frente a los posibles abusos de la producción y la distribución. De este modo se regularán producciones y sus calidades, la manipulación de productos, se deberá dar información sobre la composición, fechas de producción y de caducidad, etc.

Sin entrar en la razón por la que se desarrolla esta legislación administrativa, de lo que se dirá algo en el siguiente epígrafe, estos hechos,

33 Antonio Cancelo: «Pasado, presente y futuro...» (1992), o.c., p. 19. 
unidos a la competencia que vendrá a acomodar los precios, deberá hacer que la cooperativa de consumo profundice sobre su propio sentido en el nuevo contexto, en el que no tendrá que combatir, como en los primeros tiempos del cooperativismo, por el peso justo, la calidad adecuada y las buenas costumbres del pago al contado, lo que queda asegurado por las normas administrativas.

Al mismo tiempo la legislación cooperativa va introduciendo nuevas posibilidades en el modelo de funcionamiento de las cooperativas de consumo, a través especialmente de la venta a terceros no socios, lo que viene a superar el principio mutualista aplicado a las cooperativas de consumo.

La Ley de Cooperativas de 16 de julio de 1999 lo recoge en el art. 88.2:

Las cooperativas de consumidores y usuarios podrán realizar operaciones cooperativizadas con terceros no socios, dentro de su ámbito territorial, si así lo prevén sus Estatutos ${ }^{34}$.

\subsection{El consumidor y su defensa}

Con toda la importancia de los cambios que hemos considerado, a los que cabe añadir otros muchos en los que no hemos reparado, el cambio operado en el consumidor, que es el sujeto del consumo y que evoluciona al tiempo que todo cambia, merece la máxima atención.

Así se entendía en los III Encuentros de Cooperativas de Consumo, que organizó la Federación de Cooperativas de Consumo de Euskadi en 1992, que dedicó su temática justamente al consumidor. Francis Rigotti destacaba en aquella ocasión los cambios en los hábitos de compra debidos a la motorización, la emancipación de la mujer, el aumento de la educación y formación, así como el acceso a la información, la sensibilización ante el malgasto (que se confunde con el consumo), y la presencia de una constante innovación en los satisfactores de nuestras necesidades, junto a la desaparición de las fronteras o la globalización. En suma la presencia de un nuevo consumidor poseedor de un derecho al consumo y de un derecho a la información ${ }^{35}$.

34 En el mismo sentido el art. 105.2 de la Ley de Cooperativas de Euskadi, y en otras legislaciones.

35 Francis Rigotti: «El consumidor del 92 ¿quién es?, ¿qué será mañana?», en El consumidor del 92 (1992), pp. 12 ss. 
Aunque tal vez no se haya enfatizado suficientemente, la cooperativa de consumo, a través de los principios de Rochdale, entre los que ya se encuentran elementos característicos del consumerismo, puede considerarse la primera forma de organización histórica de los consumidores. En ella el consumidor es el que toma las decisiones y la cooperativa es el instrumento que canaliza hacia el mercado los deseos del consumidor ${ }^{36}$.

Desde principios del siglo Xx, Charles Gide, fundador de la Escuela de Nîmes juntamente con Edouard de Boyve y Auguste Fabre, había puesto de manifiesto la idea de la soberanía del consumidor en la futura «República cooperativa», punto de llegada de sus aspiraciones sociales. Se trata de «la realización de una verdadera revolución económica que haría pasar la conducción de la economía de manos de los productores a las de los consumidores» ${ }^{37}$.

Las situaciones reales van poniendo de manifiesto que los consumidores ocupan en el mercado una posición subordinada, aislados entre sí, si se compara con la que gozan los productores y distribuidores organizados, de forma que será la autoridad pública la que llevará a cabo las primeras intervenciones de defensa de la competencia y de control de precios, y que a la subordinación se suma la indefensión, no sólo en cuanto a sus intereses económicos, sino también en cuanto al cuidado de su salud frente a fraudes alimentarios y falta de control, como se ha puesto de manifiesto en los escándalos sociales que perduran en la memoria de las personas (como la talidomida o la colza, entre otros muchos) ${ }^{38}$.

Desde los años sesenta se intensifica un movimiento social en los países más desarrollados que se extendería por los países occidentales. En ese proceso tuvo importancia, con seguridad, un discurso del Presidente Kennedy en el Congreso de los Estados Unidos de América el año 1962, en el que reconocía entre otros derechos de los ciudadanos el de la protección de los intereses de los consumidores ${ }^{39}$.

EI XXIV Congreso de la Alianza Cooperativa Internacional, celebrado en Hamburgo en 1969, tomó una resolución sobre los derechos de los consumidores, según la cual éstos tienen derecho a:

1) Un nivel razonable de alimentación, vestido y vivienda.

2) Un adecuado nivel de seguridad y saludable medio ambiente libre de contaminación.

36 Antonio Cancelo: «Cooperativismo y consumerismo» (1986), o.C., pp. 194 y 211 212. También en «Posicionamiento...» (1988), o.c., p. 395; y Manuel Paniagua Zurera: Movimiento consumerista y movimiento cooperativo (1992), p. 19.

37 Alicia Kaplan de Drimer y Bernardo Drimer (1981): o.c., pp. 272 y 273.

38 Manuel Paniagua (1992): O.C., pp. 15-19.

39 Ricardo Oleaga: 25 años de consumerismo (1994), p. 17. 
3) Acceder a mercancías no adulteradas con buenos precios y una razonable variedad y opción.

4) Acceder a una información relevante sobre bienes y servicios y a educación sobre temas de consumo.

5) Influir en la vida económica y participar democráticamente en su control ${ }^{40}$.

Con estos antecedentes inmediatos nacerían en los años setenta, y se extenderían, organizaciones de los consumidores como organizaciones representativas específicas para reivindicar los derechos de los consumidores, ejercer presión ante los órganos públicos, desarrollar una legislación administrativa adecuada, facilitar información, etc. ${ }^{11}$

Con independencia de que las personas se agrupen en asociaciones de consumidores «cualquier fórmula que permita al consumidor organizarse para actuar como grupo en la sociedad resulta válida» ${ }^{42}$, y la cooperativa de consumo será un buen instrumento consumerista para sus asociados, al tiempo que la cooperativa encontrará una buena parte de su sentido especial a través de dicha acción.

\section{Un modelo particular: la cooperativa Eroski}

Eroski nació en 1969 como resultado de la fusión de nueve cooperativas de carácter local, de Vizcaya y Guipúzcoa, propiamente de siete en funcionamiento, de Eibar, Mondragón, Markina, Gernika, Amorebieta, Aretxabaleta y Ermua, y de dos nuevas que estaban en proceso de constitución, de Matiena y Recaldeberri (barrio de Bilbao) ${ }^{43}$.

En el contexto de cambio que se detectaba en los años finales de los sesenta, y en la proyección de los mismos hacia el futuro, se sentía la necesidad de superar el modelo de la pequeña cooperativa local y conseguir una dimensión que permitiera ocupar un lugar en el mercado y tener opción para conseguir los objetivos de la cooperativa en cuanto tal.

Es muy significativa, en este sentido la expresión de Antonio Cancelo: "Transformar la sociedad, aunque sólo sea un trozo de sociedad, desde el desarrollo del cooperativismo, puede quedarse en una frase

\footnotetext{
40 Turid Ström \& Kalevi Suomela: «Co-operative Consumer Policy» (1988), p. 72.

41 Ricardo Oleaga (1994): o.c., p. 18.

42 Antonio Cancelo (1986): o.c., p. 211.

43 Fermín Garmendia y Manolo González (2010): o.c., p. 30
} 
vacía de contenido si no somos capaces de hacer empresas fuertes que compitan dignamente en los mercados mundiales» ${ }^{44}$.

La dimensión deberá dar a la cooperativa ventajas desde un punto de vista empresarial a través de un mayor volumen de ventas y, consiguientemente, una capacidad de compra (y de negociación) mayor, lo que parece ser clave en el éxito empresarial del sector de la distribución.

\section{Elementos destacados del modelo}

En el nuevo modelo de cooperativa de consumo que desarrollará Eroski destacan los siguientes elementos:

- Venta a terceros y obtención de dimensión competitiva.

- Trabajadores socios de trabajo y dos comunidades que conviven y deciden conjuntamente.

- Desaparición del retorno y establecimiento de una filosofía social sobre los excedentes.

— Defensa del consumidor.

\subsection{Venta a terceros y obtención de dimensión competitiva}

Es esencial en el modelo Eroski adquirir lo que Cancelo denomina talla empresarial crítica, lo que permitirá una mejor política de compras, unificar la gama, mejorar la logística, una administración única, un mejor modelo de gestión, y, en consecuencia una mayor capacidad competitiva ${ }^{45}$, todo ello desde la consideración de que "Quedarse fuera del grupo (de las grandes empresas de distribución) es aceptar una posición de marginalidad con el consiguiente deterioro de la presencia cooperativa en el mercado $y$, lo que es peor, en la sociedad» ${ }^{46}$.

La venta a terceros dará acceso al mercado competitivo y permitirá la expansión. Esa expansión se producirá por toda la geografía nacional para formar un grupo en el que a partir de la distribución alimentaria realizan actividades de forma especializada de agencia de viajes, gasolineras, perfumería, artículos deportivos... y que cuantitativamente se puede valorar a través de los siguientes datos referidos a

\footnotetext{
44 Antonio Cancelo, «El cooperativismo ante las exigencias...» (1987): o.c., p. 89.

45 Fermín Garmendia y Manolo González (2010): o.c., p. 31.

46 Antonio Cancelo Alonso, «Pasado, presente y futuro...» (1992): o.c., p. 24.
} 
2009: 2.367 puntos de venta, 48.000 trabajadores, $2.036 .032 \mathrm{~m}^{2}$ de superficie, 661.187 socios consumidores, y una cifra de facturación de 8.427 millones de $€$ sin IVA ${ }^{47}$.

\subsection{Trabajadores socios de trabajo y dos comunidades que conviven y deciden conjuntamente}

Eroski decide que los trabajadores sean socios de trabajo y convivan plenamente con los socios consumidores. «La originalidad de Eroski, dice Constan Dacosta, ha consistido en poder integrar con un grado de eficiencia suficiente los intereses de los dos coprotagonistas de cualquier proceso productivo: el consumidor y el trabajador» ${ }^{48}$.

Esto conlleva que la mayor aportación al capital de la cooperativa la realice el trabajador, socio de trabajo, por un valor en el origen equivalente al salario de un año, siendo puramente simbólica la aportación de 100 pesetas del socio consumidor ${ }^{49}$.

La Asamblea General y el Consejo Rector son órganos participados al $50 \%$ por ambos colectivos de socios, por lo que, con independencia de la existencia de órganos específicos para los trabajadores y consumidores en el contexto de sus propios intereses y en atención a las responsabilidades que la legislación prevé para ellos, socios de trabajo y socios consumidores comparten la toma de decisiones ${ }^{50}$.

\subsection{Desaparición del retorno y establecimiento de una filosofía social sobre los excedentes}

En consecuencia con lo anterior, venta a terceros, expansión, y aportación simbólica del socio consumidor, el modelo de la cooperativa Eroski renuncia al retorno para el consumidor, siguiendo en cambio una política de fijación de un precio competitivo que lo incluya ${ }^{51}$.

En adelante los excedentes se ajustarán a una filosofía según la cual la cooperativa en cuanto empresa económica que es, conjuntamente con su condición de asociación de personas, aspira a la obtención del beneficio que justifique la bondad de su propio modelo, capaz

\footnotetext{
47 Datos facilitados por HISPACOOP, en Remedios Aceña (2010): o.c., p. 23.

48 Constan Dacosta (1991): o.C., p. 20.

49 Fermín Garmendia y Manolo González (2010): o.c., p. 49.

50 Constan Dacosta (1991): o.C., p. 21.

51 Fermín Garmendia y Manolo González (2010): o.c., p. 37.
} 
de aportar riqueza y generar empleo, y que retribuya a los trabajadores, principales actores en la consecución del resultado, con una parte del mismo, de forma que quede capitalizado sin salir de la empresa ${ }^{52}$.

Atendidos los fondos sociales, queda tradicionalmente un retorno a los socios de trabajo inferior al 50\%, de forma que salvo los fondos para la educación y promoción, de cuantías menores al 10\% que se monetizan, los demás se capitalizan. Junto al valor empresarial de tal filosofía, «queda claro para todos el enorme valor empresarial que ello tiene, pero también el sacrificio de aquellos trabajadores que renuncian a unas mejoras económicas presentes, en aras al desarrollo futuro de su organización $»^{53}$.

\subsection{Defensa del consumidor}

Constituye otro de los ejes que dan sentido al particular modelo. Tratándose de una cooperativa de consumidores, teniendo en consideración que las decisiones más importantes que afectan al consumidor, en cuanto tal, provienen del mercado, la cooperativa, en la que los consumidores participan en la toma de decisiones, les sirve de instrumento de influencia en el mismo mercado ${ }^{54}$.

En orden a este objetivo de defensa y promoción del consumidor Eroski instaló en los años setenta el laboratorio que permitiría atender los estudios que soportan una parte de la acción consumerista, y también en esos años comenzó la publicación de una revista de información al consumidor, que sumadas a la acción formativa constituyeron los comienzos del conjunto de acciones que en este orden se llevan a cabo a través de la Fundación Eroski en la actualidad.

\section{La evolución continúa}

A lo largo del tiempo se pueden considerar acciones y acontecimientos a través de los cuales Eroski ha continuado la andadura de un modelo de cooperativismo de consumo que llega a nuestros días. En el detalle de la experiencia de Eroski, nombre con el que José M. ${ }^{a}$ Arizmendiarrieta identificaba el proceso evolutivo del complejo cooperativo

52 Antonio Cancelo Alonso: «Pasado, presente y futuro...» (1992): o.c., p. 26. En última instancia, afirma, «sólo una cooperativa con beneficios podrá tener buenos precios».

53 Constan Dacosta (1991): O.C., pp. 21-22.

54 Antonio Cancelo: «Posicionamiento...»(1988), o.c., p. 395. 
de Mondragón, que resulta perfectamente aplicable a esta cooperativa $^{55}$, encontramos una pluralidad realizaciones destacables.

El paso a una dimensión regional y a la extensión por el País Vasco y Navarra es el comienzo del nuevo modelo. El afianzamiento con un acento más empresarial hizo que la cooperativa se comportara con una mayor solidez en los años ochenta, al tiempo que las cooperativas de consumo en diversos países europeos, Bélgica, Holanda, Alemania y Francia entraban en crisis ${ }^{56}$.

La experiencia internacional a finales de los años ochenta en el sur de Francia con la cooperativa Adour Pyrenées presenta una cooperativa que no teme atravesar fronteras en busca de sus objetivos ${ }^{57}$.

La expansión por España siempre tratando de encontrar una dimensión adecuada, competitiva en esta ocasión con la dimensión de las multinacionales de la distribución.

Para llevar a cabo una expansión rápida se presentaban dos grandes dificultades: por el tamaño de la expansión una dificultad financiera, por la rapidez de la misma una dificultad de formar socios cooperativistas en poco tiempo.

Es por ello por lo que la expansión se realizó bajo formas de sociedades de capitales y trabajadores por cuenta ajena, si bien el capital tenía origen cooperativo, Eroski y la cooperativa Consum de Valencia, o bien de entidades con objetivos de carácter social y comunitario, como la ONCE, Lagun-Aro (entidad de previsión de las cooperativas de la corporación Mondragón), Cajas de Ahorros, etc. sin olvidar la propia aportación de los trabajadores en el capital a través de la Gestora de participaciones GESPA.

Cabe destacar en este proceso la determinación de que los principios cooperativos debían guiar la actividad de las sociedades en las que Eroski participaba, así como la participación de los trabajadores no sólo en el capital de las citadas sociedades sino también en la gestión y en los resultados de las mismas ${ }^{58}$.

El proceso continúa en la actualidad con la adquisición en 2007 por parte de Eroski de la sociedad de distribución Caprabo de Cataluña,

55 Puede verse con detalle en Alejandro Martínez Charterina (1990): o.c., pp. 49 ss.

56 Eroski se había fijado en el complejo cooperativo Migros de Suiza, que integraba producción y distribución con buenos resultados. En el proceso Eroski trabajó con comercios asociados en franquicia, e introdujo sus marcas propias. En Antonio Cancelo: «Cooperativismo y consumerismo» (1986), o.c., pp. 201 ss., y Fermín Garmendia y Manolo González (2010): o.c., p. 96.

57 Fermín Garmendia y Manolo González (2010): o.c., p. 110.

58 Laura Gómez Urquijo y Marta de los Ríos Añón: «La sobrevivencia del modelo cooperativo en un contexto de competencia» (1999), pp. 60 a 62, y Fermín Garmendia y Manolo González (2010): o.c., pp. 124 a 129. 
con una fuerte implantación también en Madrid59, y con el deseo expreso de que todas las sociedades de Eroski puedan transformarse en cooperativas y de que todos los trabajadores puedan llegar a ser socios de las mismas ${ }^{60}$.

En el orden consumerista la actividad se canaliza a través de la Fundación Eroski. Esta «es la institución encargada de hacer efectivo el compromiso de responsabilidad social con los consumidores» ${ }^{61}$. Dicho compromiso opera hacia dentro, siendo fundamental la relación con los socios de trabajo y las características de los productos, y hacia fuera, a través de la información al consumidor, el respeto al medio ambiente y el fomento de la solidaridad local e internacional.

La página web de la Fundación Eroski62 nos permite repasar esas acciones agrupándolas en tres apartados: información al consumidor, innovación e iniciativas sociales, y desarrollo sostenible.

La información al consumidor agrupa publicaciones en papel e internet. Entre ellas la revista Consumer Eroski, mensual, con información práctica y estudios en profundidad, que se prolonga en un diario Consumer Eroski en internet, más amplio; Idea Sana Eroski para fomentar buenos hábitos de consumo, también en internet; el Barómetro de consumo anual; las Guías prácticas Consumer Eroski, verdaderas monografías especializadas; la web consumaseguridad.com sobre seguridad alimentaria...

La información se completa con las acciones formativas a través de las Escuelas, también on line, a las cuáles se convoca desde las distintas publicaciones.

La innovación e iniciativas sociales recogen, por el lado de la innovación, el laboratorio de análisis como instrumento al servicio de estudios e investigación, así como las becas de investigación que se conceden, y, por el lado de las iniciativas sociales la participación en organizaciones no gubernamentales y las diversas campañas que se promocionan, comercio justo, recogida de ropa, etc. Dentro de este apartado se apoya y fomenta la diversidad cultural de las localidades en las que se enmarca la acción empresarial.

59 «Grupo Eroski adquiere Caprabo», noticia de 6/06/2007, en www.eleconomista. es/empresas-finanzas/noticias/224708/06/07/Grupo-Eroski-adquiere-Caprabo.html

60 «Eroski quiere hacer cooperativistas a todos sus empleados, incluidos los de Caprabo», noticia de 2/0472008, en www.cincodias.com/articulo/empresas/eroski-quierehacer-cooperativistas-todos-empleados-incluidos-caprabo/20080402cdsdiemp_1

61 Marta Aréizaga Ezcurdia: «Responsabilidad social con los consumidores» (2002), p. 2.

62 www.fundacioneroski.es/web/es 
Por fin, el desarrollo sostenible ampara la protección del medio ambiente mediante acciones ecológicas en los puntos de venta relacionadas, por ejemplo con el uso de bolsas reutilizables, o campañas de sensibilización variadas, programas de ayuda a iniciativas de medio ambiente y entorno local, y proyectos de desarrollo sostenible en el tercer mundo.

\section{Alternativas}

El modelo de Eroski para llevar a cabo un cooperativismo de consumo en un contexto de cambio en el que muchas cooperativas de consumo de muchos países han desaparecido, como es natural, no es un modelo único, toda vez que si unas han desaparecido otras han sobrevivido y otras nuevas se han constituido. Hay cooperativas de consumo que siguen modelos más tradicionales, participando unas y otras de la consideración de que una cooperativa es una asociación de personas que deciden poner al servicio de una necesidad compartida por ellas una empresa económica que les ayude a satisfacer esa necesidad.

Tal vez porque se está poniendo de moda, al menos porque se empieza a hablar de ella63, haré un comentario de la Park Slope Food Coop, situada en el barrio del mismo nombre de Brookling, Nueva York.

Se trata de la cooperativa de consumidores con una única tienda más grande de los Estados Unidos de América por su cifra de ventas (39,4 millones de dólares en 2009).

Fundada en 1973, vende exclusivamente a sus 15.100 socios consumidores (para serlo han de depositarse 100 \$ y aportar 25 más, así como participar en una sesión introductoria), los cuales prestan 2 horas y 45 minutos de trabajo cada cuatro semanas (almacenamiento, preparación de productos para la venta, labores administrativas, atención a la guardería...), alrededor del $75 \%$ del trabajo de la cooperativa, de forma que ésta funciona con sólo 65 empleados a tiempo completo ${ }^{64}$.

63 Es conocida su posición contra la venta de productos de Coca-Cola que fue aprobada en Asambea General en 2004, contenida en la «Resolución para remover los productos de Coca-Cola de los estantes de la Park Slope Food Coop» por tratarse de productos contrarios a sus Estatutos que piden «evitar los productos que dependan de la explotación de los demás». En www.killercoke.org/resolution_psfcres.php

64 Datos ofrecidos por Beth Kowitt, "The rise of the grocery co-op» (19 de septiembre de 2010), en money.cnn.com/2010/09/16/news/companies/grocery_coop_Brooklyn. fortune/index.htm 
Utiliza un inventario de sólo 9.500 productos de la mayor parte de las categorías de alimentos (frente a más de 50.000 de un centro equivalente) en una superficie de 6.000 pies cuadrados, con una consiguiente rotación muy alta de artículos.

Se estima el ahorro de los compradores entre un 20 y un $40 \%$, los cuales reciben mucha información, a través de una gestión transparente, sobre los proveedores y el origen de los productos.

La misión de Park Slope Food Coop 65 , recoge estas ideas, la contribución al trabajo, que genera confianza y permite mantener los precios más bajos, la compra exclusiva por los socios que comparten responsabilidad y beneficio. Se consideran parte activa del movimiento cooperativo.

Entre la variedad de productos que ofrecen ponen un acento en los ecológicos, mínimamente procesados y saludables. Respetan el medio ambiente. Tienen preferencia en sus compras los productores locales. Reciclan. Y potencian la educación acerca de todos estos temas.

Se sienten comprometidos con la diversidad y la igualdad, y tratan de maximizar la participación.

A través de su página web se puede acceder a la información que ofrece, que llega a facilitar la relación de sus proveedores, el origen de los productos, su publicación periódica quincenal The Linewaiters' Gazette, así como la relativa a sus actividades y campañas ${ }^{66}$.

Unas doscientas cooperativas están iniciando su andadura siguiendo este modelo de cooperativa local, de barrio o de buena vecindad.

\section{Conclusión}

Para concluir este trabajo apuntaré que las cooperativas de consumo en sus casi dos siglos de existencia han sufrido la evolución correspondiente a un tiempo cambiante con una intensidad y una velocidad como nunca antes en la historia lo había sido.

Han tenido que acomodarse y encontrar su sitio en cada momento y han desarrollado diversos modelos, de los que he presentado con más detenimiento el de la cooperativa Eroski, no sólo porque me resulta más próximo, conocido y estimado, sino también por su relieve y

65 Contenida en la página web de la cooperativa, foodcoop.com

66 Actividades como los servicios de guardería para los niños de los socios que están trabajando o comprando, talleres, y actos diversos, así como campañas para la promoción del reciclado, y otras. 
sus consecuciones que han puesto a la cooperativa de consumo a la altura de las sociedades más importantes en su sector.

Pero también he acercado un modelo tan actual y al mismo tiempo tan tradicional como la Park Slope Food Coop.

El cooperativismo de consumo continúa teniendo su espacio y su misión, hoy vinculado al consumerismo, a la formación y defensa del consumidor, y a la conservación de nuestro medio, pero siempre alrededor de los dos ejes que constituyen la esencia de la cooperativa, la democracia y la participación ${ }^{67}$.

\section{Bibliografía}

ACEÑA, Remedios: "Cooperativas y consumidores: Los protagonistas de estos veinte años», en Cuadernos de las cooperativas de consumidores, n. ${ }^{\circ} 19$, HISPACOOP, Madrid, junio de 2010, pp. 8 a 25.

ACl: Declaración de la Alianza Cooperativa Internacional sobre la Identidad Cooperativa, Consejo Superior de Cooperativas de Euskadi, Vitoria-Gasteiz, 1996.

AréIZAGa EZCURDIA, Marta: «Responsabilidad social con los consumidores. La misión de la Fundación Grupo Eroski», en Compromiso social y transparencia en las cooperativas de consumidores. Documentos de trabajo. Confederación española de cooperativas de consumidores y usuarios. Madrid, 2002. Es la tercera ponencia.

ARRIETA, Leyre et al.: El movimiento cooperativo en Euskadi (1884-1936), Fundación Sabino Arana-Mondragón Corporación Cooperativa, Bilbao, 1998.

BAYON, Denis: Le commerce véridique et social de Michel-Marie Derrion (Lyon, 1835-1838). Atelier de Création Libertaire, Lyon, 2002.

Cancelo Alonso, Antonio: "Cooperativismo y consumerismo», en Anuario de Estudios Cooperativos 1986, Universidad de Deusto, Bilbao, pp. 193 a 228.

— : «El cooperativismo ante las exigencias del mercado mundial», en Anuario de Estudios Cooperativos 1987, Universidad de Deusto, Bilbao, pp. 81 a 89.

67 En este sentido se posicionaban Ström y Suomela: o.c., p. 76, y éste resultaba ser el tema de las Jornadas Europeas organizadas por Hispacoop en junio de 2010 en Barcelona "La participación del socio consumidor», tras reconocer el crecimiento económico y el aumento del número de socios. "Este crecimiento y progresivo aumento de su base social ha traído como consecuencia, en determinados casos, el paulatino debilitamiento del sentimiento de pertenencia del socio a la experiencia cooperativa y por tanto una menor participación del socio en la actividad cooperativa», en "Motivar la participación del socio consumidor en las grandes cooperativas de consumo» (2010), p. 43. 
— : «Posicionamiento del cooperativismo de consumidores vasco», en I/ Congreso mundial vasco. Congreso de cooperativismo. Universidad de DeustoGobierno Vasco, Bilbao, 1988, pp. 391 a 396.

- : «Pasado, presente y futuro del cooperativismo de consumo en España», en Anuario de Estudios Cooperativos 1992, Universidad de Deusto, Bilbao, pp. 13 a 27.

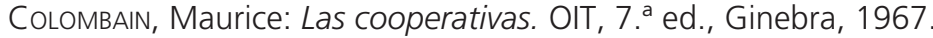

"Consumer and User Co-operatives in Spain», en Review of International Cooperation, vol. 83, n. ${ }^{\circ}$ 2, I.C.A., Geneva, 1990, pp. 6 a 8.

DACOSTA, Constan: «Eroski, una adaptación al cambio», en Anuario de Estudios Cooperativos 1991, Universidad de Deusto, Bilbao, pp. 17 a 29.

Delom, Bernardo: «Introducción», en HolyoAKe, Jorge Jacobo. Historia de los pioneros de Rochdale, AECOOP-Aragón, Zaragoza, 1973, pp. 11 y 12.

FAURA Ventosa, Ignasi y UdinA, Teresa: "La actividad de las cooperativas de consumidores», en Economía social. La actividad económica al servicio de las personas, Cajamar Caja Rural, s.l., s.f., pp. 146 a 159. El texto es de www. fundacioncajamar.com/mediterraneo/revista/me0609.pdf

GaRmendia, Fermín y GonzÁlez, Manolo: Principios, pensamiento e innovación empresarial. La visión de Antonio Cancelo. Esic Ed., Madrid, 2010.

HISPACOOP: Cooperativas de consumidores y usuarios. Cómo empezar, Barcelona, 2001.

GÓmez URQUIJO, Laura y Ríos AÑón, Marta de los: «La sobrevivencia del modelo cooperativo en un contexto de competencia», en Revista de la Cooperación Internacional, vol. 32, n. ${ }^{\circ}$ 1/1999, A.C.I., INTERCOOP, Buenos Aires, pp. 55 a 66.

HolyOAKE, George Jacob: Historia de los pioneros de Rochdale, Intercoop, Buenos Aires, 1989.

Kaplan de Drimer, Alicia y Drimer, Bernardo: Las cooperativas. Fundamentos,

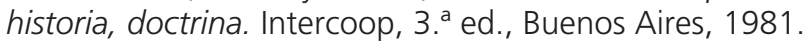

LAMBert, Paul: La doctrina cooperativa. Intercoop, 3. ${ }^{a}$ ed. en español, Buenos Aires, 1970.

Laws and objets of the Rochdale Society of Equitable Pioneers: enrolled according to the Acts, $10^{\text {th }}$, George $I V$, and $4^{\text {th }}$ and $5^{\text {th }}$, William IV. Printed by Jesse Hall, Rochdale, 1844

Martínez ChARTERINA, Alejandro: Análisis de la integración cooperativa, Universidad de Deusto, Bilbao, 1990.

- : "La cooperativa como empresa y los principios cooperativos tras el congreso centenario de la $\mathrm{ACl}$ de Manchester», en Evolución del escenario económico, Universidad de Deusto, Bilbao, 1996, pp. 207 a 226.

Mladenatz, Gromoslav: Historia de las doctrinas cooperativas. Intercoop, Buenos Aires, 1969.

«Motivar la participación del socio consumidor en las grandes cooperativas de consumo», en Cuadernos de las cooperativas de consumidores, n. ${ }^{\circ} 20$, HISPACOOP, Madrid, octubre de 2010, pp. 43 a 47.

OleAgA, Ricardo: 25 años de consumerismo, Eroski, Elorrio, 1994. 
PANIAGUa ZURERA, Manuel: Movimiento consumerista y movimiento cooperativo, Ministerio de Sanidad y Consumo, Madrid, 1992.

RIGOTII, Francis: «El consumidor del 92 ¿quién es?, ¿qué será mañana?», en El consumidor del 92, Federación de Cooperativas de Consumo de Euskadi, Vitoria-Gasteiz, 1992, pp. 5 a 19.

Roussell, Concepción y Alboniga, Roberto: Historia de las cooperativas de consumo vascas, Federación de Cooperativas de Consumo de Euskadi, VitoriaGasteiz, 1994.

STRÖM, Turid \& SUOMELA, Kalevi: «Co-operative Consumer Policy», en Review of International Co-operation, vol. 81, n. ${ }^{\circ}$ 2, I.C.A., Geneva, 1988, pp. 71 a 76.

Trujlllo Díez, Iván Jesús: Cooperativas de consumo y cooperativas de producción, Aranzadi, Elcano (Navarra), 2000. 